\title{
Similar patterns of 18F-FDG brain PET hypometabolism in paediatric and adult patients with long COVID: a paediatric case series
}

\section{Aurelie MORAND}

AP-HM: Assistance Publique Hopitaux de Marseille Jacques-Yves CAMPION

AMU: Aix-Marseille Universite

Anne LEPINE

AP-HM: Assistance Publique Hopitaux de Marseille

\section{Emmanuelle BOSDURE}

AP-HM: Assistance Publique Hopitaux de Marseille

\section{Léa LUCCIANI}

AP-HM: Assistance Publique Hopitaux de Marseille

\section{Serge CAMMILLERI}

AMU: Aix-Marseille Universite

\section{Brigitte CHABROL}

AMU: Aix-Marseille Universite

Eric Guedj ( $\nabla$ eric.guedj@ap-hm.fr )

CHU Timone https://orcid.org/0000-0003-1912-6132

\section{Research Article}

Keywords: SARS-CoV-2, complaints, cognition, fatigue, children

Posted Date: July 19th, 2021

DOI: https://doi.org/10.21203/rs.3.rs-722537/v1

License: (c) (1) This work is licensed under a Creative Commons Attribution 4.0 International License.

Read Full License

Version of Record: A version of this preprint was published at European Journal of Nuclear Medicine and Molecular Imaging on August 19th, 2021. See the published version at https://doi.org/10.1007/s00259021-05528-4. 


\section{Abstract}

Purpose. Several weeks after COVID-19 infection, some children report the persistence or recurrence of functional complaints. This clinical presentation has been referred as "long COVID" in the adult population, and an ${ }^{18} \mathrm{~F}$-FDG brain PET hypometabolic pattern has recently been suggested as a biomarker. Herein, we present a retrospective analysis of 7 paediatric patients with suspected long COVID who were explored by ${ }^{18} \mathrm{~F}-\mathrm{FDG}$ brain PET exam. Metabolic brain findings were confronted to those obtained in adult patients with long COVID, in comparison to their respective age-matched control groups.

Methods. Review of clinical examination, and whole-brain voxel-based analysis of ${ }^{18} \mathrm{~F}$-FDG PET metabolism of the 7 children in comparison to 20 paediatric controls, 35 adult patients with long COVID and 44 healthy adult subjects.

Results. Paediatric patients demonstrated a similair brain hypometabolic pattern as that found in adult long COVID patients, involving bilateral medial temporal lobes, brainstem and cerebellum ( $p$-voxel < 0.001 , p-cluster $<0.05$ FWE-corrected), and also the right olfactory gyrus after small volume correction ( $p$ voxel $=0.010 \mathrm{FWE}$-corrected), with partial recovery in two children at follow-up.

Conclusion. These results provide arguments in favour of possible long COVID in children, with a similar functional brain involvement to those found in adults.

\section{Introduction}

Sequelae of COVID-19 have been described following SARS-CoV-2 infection in patients with pulmonary and extrapulmonary lesions ${ }^{1,2}$. In addition to those subjects usually identified by conventional explorations, others report persistent or delayed functional complaints beyond 3-4 weeks after the onset of the disease in the absence of obvious morphological lesions ${ }^{3-6}$. This last group of patients with long COVID mostly includes younger subjects with mild to moderate initial presentation ${ }^{7}$. Current recommendations specify that a positive biological confirmation of SARS-CoV-2 is not required to identify these patients ${ }^{8-10}$. Indeed, many of these patients with mild to moderate, or even asymptomatic, initial presentation have not been tested by RT-PCR at the acute stage, or at least not at the time of high viral load excretion, and cases with negative serology have also been described, especially those involving younger patients ${ }^{11-14}$. In this context, we previously identified a brain PET hypometabolism in adult patients with long COVID, involving the olfactory bulb, and the brain network connected to this region, in particular the temporal lobe, the brainstem, and the cerebellum ${ }^{15-17}$. This brain hypometabolism had value to discriminate individual patients from healthy subjects, with more severe hypometabolism found in patients with more numerous complaints.

While long COVID is currently being recognized by the medical community $4,5,8-10$, the paediatric forms are still debated. However, if long COVID mostly affects younger patients with few initial symptoms ${ }^{15,16}$, the occurrence of such presentations in paediatric patients should be investigated. Such presentations could 
indeed have significant healthcare and social implications. In this line, two published case reports from Sweden and Latvia first described long COVID in few children ${ }^{18,19}$. More recently, an Australian study reported post-acute COVID-19 symptoms in $8 \%$ of 151 children followed more than 3 months after having been tested positive for SARS-CoV- $2^{20}$. Moreover, an Italian study reported that more than $50 \%$ of a cohort of 129 children had residual symptoms at follow up 4 months after the initial diagnosis ${ }^{21}$. And in a preprint study from United Kingdom ${ }^{22}, 4.4 \%$ of 1,734 children who were positively tested for SARS-CoV2 experienced illness duration $\geq 28$ days.

Here, we present the clinical description of 7 paediatric cases with symptoms suggesting long COVID, who were initially explored with brain ${ }^{18} \mathrm{~F}-\mathrm{FDG}$ PET, with also two follow-up exams. The pattern of hypometabolism, obtained in comparison to 21 paediatric controls, was confronted to those previously reported between adult patients with long COVID and adult healthy subject ${ }^{16}$.

\section{Methods}

\section{Subjects}

Seven consecutive paediatric patients with symptoms suggesting a long COVID, who were explored by ${ }^{18} \mathrm{~F}-\mathrm{FDG}$ brain PET, were retrospectively included in our centre at Timone Hospital, Marseille, France, from August 20th, 2020 to November 17th, 2020. The clinical criteria of inclusion were the following: children under 18 years old, with an history compatible with SARS-CoV-2 infection, having had a medical evaluation 4 weeks or more after initial symptoms for functional complaints (persistent or recurrent fatigue, fever, chills, anorexia, weight loss, bulimia, dyspnoea, cough, headache, chest pain, musculoskeletal pain, diarrhoea, haematuria, dysmenorrhea, skin rash, dysautonomia such as palpitations and vagal hypotension, hyposmia/anosmia, dysgeusia/ageusia, cognitive impairment such as memory difficulties and concentration difficulties, hypersomnia, insomnia, stress, depression, visual impairments, decreases in scholastic achievement and limitation of extracurricular activities). During this inclusion period of less than 3 months, and given the weak data then available in the literature on long COVID, the aetiology of such clinical presentations was considered particularly uncertain. The PET exam was performed to investigate differential diagnosis and particularly possible encephalitis, whose presentation can be particularly atypical in children ${ }^{23}$. No other PET exam was performed in this context from November 17th, 2020 to the submission of the present article (except the control of two of the 7 children thereafter presented). Of note, our centre is a paediatric hospital with strong experience of brain ${ }^{18} \mathrm{~F}$-FDG PET in this population.

Confirmed SARS-CoV-2 infection was defined by a positive biological result established by RT-PCR at the time of initial symptoms or by serology (ELISA or immune assay). Probable SARS-CoV-2 infection was defined by clinical features during the pandemic period (one or several of the following: fever, cough, dyspnoea, chest pain, pneumoniae, asthenia, headache, rhinitis, pharyngitis, muscular pain, diarrhoea, 
abdominal pain, hyposmia/anosmia, dysgeusia/ageusia). Contact with a confirmed case was searched for these patients.

We collected the clinical and paraclinical variables from the electronic patient record system (DPI aXigate $\left.{ }^{\circledR}\right)$ : age; sex; history of contact with a proven COVID-19 case before symptoms; day 0 of SARSCoV-2 infection as defined by initial symptom onset; details of initial symptoms; initial level of C-reactive protein; initial clinical general status, described as asymptomatic, mild, moderate or severe according to Dong et al. ${ }^{24}$ and using NEWS score ${ }^{16}$; initial CT lung severity score (using 2 grades: normal/minimal vs. intermediate/severe involvement); co-infection; SARS-CoV2 RT-PCR; SARS-CoV2 serology; initial treatment (antibiotics, other); long-course treatment before acute COVID-19; possible differential diagnosis; and functional complaints more than 4 weeks after acute COVID-19.

At comparative purpose, we selected age-matched control patients $(n=20$; mean age 12.7yrs \pm 2.2$)$ scanned under identical conditions from our database, in whom visual interpretation was normal and somatic cerebral diseases thereafter excluded at the follow-up. PET findings obtained between these two paediatric groups (long COVID and controls), were compared to those previously reported in adult long COVID and healthy subjects of our previous article ${ }^{16}$.

This data collection in adult and paediatric patients was retrospective, requiring no ethical approval after written inquiry and response from our institutional board (registration of the response on the following reference: PADS20-296). The patients and their parents or legal representatives received information and were not opposed to the use of their anonymized medical data in accordance with French and European regulations. This study respects the GPDR (General Data Protection Regulation) requirements.

Data in adult healthy subjects correspond to a previous interventional study registered in ClinicalTrials.gov with the following reference NCT00484523, and ethically approved by CPP Sud Mediterranée on the following reference 2007-A00180-53.

\section{${ }^{18}$ F-FDG PET imaging and processing}

${ }^{18} \mathrm{~F}-\mathrm{FDG}$ brain PET scans were acquired in the same centre using a same protocol, in subjects fasted for at least 4 hours with a controlled, normal glycaemia level, using an integrated PET/CT General Electric camera (Waukesha, WI), after intravenous administration of $111 \mathrm{MBq}$ per 15-min acquisition at $30 \mathrm{~min}$ post-injection. Images were reconstructed on a 192×192 matrix using the ordered subsets expectationmaximization algorithm and corrected for attenuation using a CT transmission scan.

The SPM8 software (Wellcome Department of Cognitive Neurology, University College, London, UK) was used to proceed whole-brain voxel-based analysis. The four groups of subjects were implemented in a same ANOVA statistical model, with age and sex as nuisance covariables. In detail, the PET images were spatially normalized using the Montreal Neurological Institute (MNI) atlas. The dimensions of the resulting voxels were $2 \times 2 \times 2 \mathrm{~mm}$. The images were smoothed with a Gaussian filter ( $8 \mathrm{~mm}$ full-width at half-maximum) to blur individual variations in the gyral anatomy and to increase the signal-to-noise ratio. 
Proportional scaling was performed to give the same global metabolic value to each PET examination. The SPM(T) PET maps were obtained at a height threshold (voxel-level significance) of $p<0.001$, with a correction for multiple comparison at cluster-level using the family-wise error (FWE) rate for a p-cluster < 0.05. The whole-brain statistical comparison was first performed between paediatric patients with long COVID and paediatric controls, to search hypo- or hypermetabolism in patients. In case of no significant findings at this whole-brain correction level for one or more of the four regions previously identified as hypometabolic in adult patients with long Covid (olfactory gyrus, medial temporal lobe, brainstem, cerebellum), this approach was completed by a small volume correction on regions-of-interest defined on AAL atlas ${ }^{25}$ ( $p$-voxel $<0.05$ FWE-corrected). Finally, the comparison was done at whole-brain voxel-based level between the four groups of subjects to identify regions which were possibly more hypometabolic in adult patients than in paediatric patients with long COVID (in comparison to their respective controls groups), and those which were more hypometabolic in paediatric patients than in adult patients with long COVID (1-1 -1 1 and - 1 11-1 contrasts, considering the four groups on the following order: children with long COVID, paediatric controls, adults with long Covid, healthy adult subjects).

\section{Results}

\section{Characteristics of the paediatric patients with long COVID At the acute stage}

Between February 25th, 2020, and October 10th, 2020, 11,074 patients under 16 years old were biologically tested for COVID-19 in our hospital (with 661 positive tests). Among them, 7 afterwards reported persistent symptoms and were explored by ${ }^{18} \mathrm{~F}$-FDG PET.

The characteristics of the 7 children are detailed at group-level in Table 1, and at individual-level in Supplementary Table 1. The mean and median age was 12 years old (10-13 years), with 6 girls and one boy. All had initial symptoms compatible with COVID-19, 6 during the first wave of the pandemic in France between February and March 2020, and the last one during the second wave in October 2020. They presented moderate initial presentation, with a NEWS score between 0 and 1 . The main common symptoms were fever, muscular pain, asthenia, rhinitis, hyposmia/anosmia and dysgeusia/ageusia. 
Table 1

Acute phase COVID-19 and long Covid symptoms

\begin{tabular}{|c|c|}
\hline \multicolumn{2}{|c|}{ Characteristics of children with long COVID symptoms } \\
\hline $\mathrm{N}$ & 7 \\
\hline Sex & 6 girls \\
\hline Age in year: Mean [Min, Max] & $12[10-13]$ \\
\hline \multicolumn{2}{|c|}{ Acute COVID-19 clinical and paraclinical symptoms } \\
\hline Fever & $6 / 7$ \\
\hline Cough & $3 / 7$ \\
\hline Dyspnoea & $3 / 7$ \\
\hline Chest pain & $0 / 7$ \\
\hline Pneumoniae & $0 / 7$ \\
\hline Asthenia & $5 / 7$ \\
\hline Headache & $3 / 7$ \\
\hline Rhinitis & $5 / 7$ \\
\hline Pharyngitis & $1 / 7$ \\
\hline Muscular pain & $6 / 7$ \\
\hline Diarrhoea & $2 / 7$ \\
\hline Abdominal pain & $1 / 7$ \\
\hline Hyposmia/anosmia & $5 / 7$ \\
\hline Dysgeusia/ageusia & $5 / 7$ \\
\hline High level of CRP & $0 / 3$ \\
\hline CT lung abnormalities & $0 / 1$ \\
\hline NEWS score: Median [Min, Max] & $1[0,1]$ \\
\hline Contact with previous proven COVID-19 case & $3 / 7$ \\
\hline SARS-CoV-2 infection confirmation & $3 / 7$ \\
\hline Positive SARS-CoV-2 RT-PCR & $1 / 5$ \\
\hline Positive SARS-CoV-2 serology & $2 / 6$ \\
\hline Initial treatment & 3 \\
\hline
\end{tabular}




\begin{tabular}{|ll|}
\hline Characteristics of children with long COVID symptoms & \\
\hline Hydroxychloroquine & 0 \\
\hline Antibiotics (Clindamycine, Azythromycine) & $3(1,2)$ \\
\hline Systemic corticosteroids & 1 \\
\hline Nasal decongestant & 0 \\
\hline Long COVID symptoms & 7 \\
\hline Fatigue & $5 / 7$ \\
\hline Fever/Hyperthermia & $3 / 7$ \\
\hline Chilliness & $1 / 7$ \\
\hline Anorexia & $3 / 7$ \\
\hline Significative weight loss & $2 / 7$ \\
\hline Bulimia & $1 / 7$ \\
\hline Dyspnoea & $4 / 7$ \\
\hline Cough & $3 / 7$ \\
\hline Musculoskeletal pain & $3 / 7$ \\
\hline Headache & $3 / 7$ \\
\hline Diarrhoea & $3 / 7$ \\
\hline Skin rash & $3 / 7$ \\
\hline Dysautonomic symptoms (palpitations and Vagal malaise) & $1 / 7$ \\
\hline Visual impairment & $1 / 7$ \\
\hline Hyposmia/anosmia & $3 / 7$ \\
\hline Dysgeusia/ageusia & $1 / 7$ \\
\hline Cognitive impairment & $3 / 7$ \\
\hline Hypersomnia & $3 / 7$ \\
\hline Insomnia & $3 / 7$ \\
\hline Stressed felling & $3 / 7$ \\
\hline Decrease of scholar implication & $3 / 7$ \\
\hline Limitation of extrascholar activities & $3 / 7$ \\
\hline
\end{tabular}


Biological confirmation of SARS-CoV-2 infection was available for 3 of the 7 children. SARS-CoV-2 RTPCR was positive in one of the 5 patients for whom the test was performed. SARS-CoV-2 serology was positive for 2 of the 6 tested children. For 4 of the 7 children without biological confirmation, the initial symptoms were highly suggestive of COVID-19, and $3 / 4$ had contact with a biologically demonstrated case.

Three children were treated with antibiotics ( 2 with azithromycin and 1 with clindamycin). One received systemic corticosteroids.

\section{At the long COVID stage}

The 7 children had persistent symptoms for more than 4 weeks following the initial acute COVID-19 symptoms, without symptom-free interval. The main reported symptoms were fatigue and cognitive impairment such as memory and concentration difficulties.

Three of the 7 children had a brain MRI, with nonspecific FLAIR hypersignal of right caudate nucleus in child \#3, and no abnormalities in child \#4 and \#7. Concerning child \#2, anti-self-DNA antibodies were found during the explorations, but there was not sufficient evidence to diagnose an autoimmune disease. Cytomegalovirus co-infection and positive serology (both $\lg \mathrm{M}$ and $\lg \mathrm{G}$ ) were found in child \#7.

The mean and median delay between initial symptoms and the PET exam was 5 months (1-8 months). Child \#4 and child \#7 received a second PET exam on the follow-up, respectively, at 12 and 6 months after the acute phase, while clinical presentation was partially improved for child \#4 and unchanged for child \#7. PET imaging were partially improved for both, especially for the brainstem (Fig. 1; see below).

\section{${ }^{18}$ F-FDG brain PET findings}

Figure 1 presents a selection of individual ${ }^{18} \mathrm{~F}-\mathrm{FDG}$ brain PET slices of each of the 7 children, and the PET follow-up for children \#4 and \#7. An example of normal PET exam is also added from one child of 10 years old of the paediatric control group in this Fig. 1.

In comparison to paediatric controls, children with long COVID exhibited hypometabolism in bilateral medial lobes (amygdala, uncus, parahippocampal gyrus), the pons and cerebellum ( $\mathrm{p}$-voxel $<0.001, \mathrm{p}$ cluster < 0.05 FWE-corrected; Fig. 2 and Table 2). Hypometabolism of the right olfactory gyrus was also confirmed using small volume correction on anatomical region-of-interest from AAL atlas ( $p$-voxel $=0.010$ FWE-corrected), and with a trend for the left olfactory gyrus ( $p$-voxel $=0.058$ FWE-corrected). No significant hypermetabolism was found in paediatric patients in comparison to controls. 
Table 2

Main ${ }^{18} \mathrm{~F}$-FDG PET hypometabolism in paediatric patients with long COVID in comparison to paediatric controls (p-voxel $<0.001$, p-cluster $<0.05$ FWE corrected), including age and sex in the statistical model.

\begin{tabular}{|c|c|c|c|c|c|}
\hline \multirow{2}{*}{$\begin{array}{l}\text { cluster } \\
\text { k }\end{array}$} & \multirow{2}{*}{$\begin{array}{l}\text { voxel } \\
\text { T-score }\end{array}$} & \multicolumn{3}{|c|}{ Talairach coordinates } & \multirow[t]{2}{*}{ Localization } \\
\hline & & $\mathbf{x}$ & y & $\mathbf{z}$ & \\
\hline \multirow[t]{10}{*}{8,835} & 5.11 & 32 & 1 & -20 & Right Uncus \\
\hline & 4.83 & -28 & -15 & -21 & Left Parahippocampal Gyrus \\
\hline & 4.58 & -28 & -47 & -40 & Left Cerebellar Tonsil \\
\hline & 4.56 & 14 & -48 & -28 & Right Cerebellum \\
\hline & 4.45 & -8 & -29 & -31 & Pons \\
\hline & 4.32 & -24 & -3 & -18 & Left Amygdala \\
\hline & 4.29 & -14 & -66 & -39 & Left Inferior Semi-Lunar Cerebellar Lobule \\
\hline & 4.25 & 28 & -28 & -15 & Right Parahippocampal Gyrus \\
\hline & 4.18 & 18 & -64 & -39 & Right Inferior Semi-Lunar Cerebellar Lobule \\
\hline & 4.13 & 24 & -35 & -34 & Right Cerebellar Tonsil \\
\hline
\end{tabular}

The $k$-value represents the number of voxels inside a particular cluster. Talairach coordinates are expressed in mm. BA: Brodmann Area

No statistical difference was found between hypometabolism of paediatric patients with long COVID (obtained in comparison to paediatric controls) and those of adult patients with long COVID (obtained in comparison to healthy subjects) using two-sided contrasts (no more hypometabolism in adults or in children; p-voxel < 0.001, p-cluster <0.05 FWE-corrected).

\section{Discussion}

This case series presents 7 children with persistent functional complaints after proven or suspected SARS-CoV-2 infection from a single centre with evidence of a PET hypometabolism pattern similar to that previously found in adults with long COVID, involving the olfactory gyrus and medial temporal lobes extending to the pons and cerebellum ${ }^{16}$. These findings provide arguments in favour of possible long COVID in children, with a common functional brain involvement among subjects regardless of age. The number of 7 children with such presentation has to be considered relative to the number of children with a positive diagnosis of SARS-CoV-2 in our institution during the same global period $(n=661)$.

Evidence of long COVID in children and adolescents remains scarce in the literature. Several studies have suggested a presentation similar to that reported in adults, with persistent and recurrent functional complaints. In a published case series, Lugvigson et al ${ }^{18}$ reported five Swedish children (median age of 
12 years, range $9-15$, four girls and one boy) with long COVID symptoms $6-8$ months after initial suspected COVID-19 infection diagnosed by their physician. However, none of these patients had recent positive SARS-CoV-2 tests (PCR or serology). Smane et al. ${ }^{19}$ described 9/30 Latvians children of the same centre suffering from persistent symptoms after a confirmed SARS-CoV-2 infection with mild to moderate illness, whereas their PCR had become negative. On the other hand, Say et al ${ }^{20}$ reported postacute COVID-19 symptoms in 12/151 Australian children more than 3 months after having been tested positive for SARS-CoV-2 (median age of 2 years, range 1-7, five girls, seven boys). Moreover, Buonsenso et al. ${ }^{21}$ described preliminary evidence of long COVID in 68/129 Italian children, 120 days after they were diagnosed with confirmed SARS-CoV-2 infection. Finally, in a preprint Molteni et al. ${ }^{22}$ reported the results of a comparative study from United Kingdom. Of the 1,734 children tested positive for SARS-CoV2, 4.4\% experienced illness duration $\geq 28$ days after the acute symptoms. These findings were significatively more frequent in older children (12-17 years old) than in younger ones (5-11 years old), $5.1 \%$ vs. $3.1 \%$, and more frequent than in the negative-tested cohort $(0.9 \%$ of 1,734 children matched for age, gender, and week of testing).

Our study is the first to identify brain PET hypometabolism in children suspected of having long COVID. Further studies will be needed to determine whether this pattern could be used as a biomarker to help with the positive or differential diagnosis of severe or atypical forms of long COVID, especially in children, in whom acute COVID-19 diagnosis is more difficult and often not biologically proven ${ }^{11-14}$. It could also be useful during the follow-up to evaluate recovery, as suggested in children \#4 and \#7 of our series.

Several hypotheses have been proposed and still investigated to explain long COVID. These include, but are not limited to, inflammatory or viral neurotropism ${ }^{1,3,26-29}$ from persistent sites of infection, particularly from the olfactory pathways ${ }^{30-33}$, dysimmune reaction ${ }^{34}$, vascular abnormalities, disturbances of gut-brain relationships ${ }^{35}$, and possible interactions with psychological factors.

This case-series is limited by the number of children that are reported, as previous few published studies in paediatric long COVID. The absence of healthy paediatric subjects is another obvious limitation. This healthy group is nevertheless difficult to establish, from an ethical point of view, and findings were obtained in comparison to age-matched control patients scanned under identical conditions, in whom visual interpretation was normal and somatic cerebral diseases excluded. Moreover, previous statistical reports using the same statistical parametric mapping approach have demonstrated that children over 6 years of age appear to display the same pattern of glucose utilization as adults, with spatial normalization of brain PET of adult template also possible without significant artefacts ${ }^{36}$. Further studies will be finally needed to specify the longitudinal sequence of brain metabolic abnormalities ${ }^{29}$.

\section{Conclusion}

These results provide arguments supporting the possibility of long COVID in children, with functional brain metabolism patterns similar to those found in adult patients. Further studies are needed to improve 
the understanding of this new clinical entity, especially its exact pathophysiology, the possible recovery on follow-up, and the overall epidemiology, including the genuine frequency of such presentation in the paediatric population.

\section{Declarations}

\section{Disclosure}

Authors have nothing to disclose.

\section{Funding}

The prospective part of the study on healthy subjects was funded by APHM.

\section{Acknowledgements:}

We thank Drs Violaine Bresson, Charlotte Pouchoux, Diego Urbina, Carole Eldin, and Elsa Kaphan for their clinical feedback.

\section{References}

1. Kas A, Soret M, Pyatigoskaya N, Habert MO, Hesters A, Le Guennec L, et al. The cerebral network of COVID-19-related encephalopathy: a longitudinal voxel-based 18F-FDG-PET study. Eur J Nucl Med Mol Imaging. 2021. https://doi.org/10.1007/s00259-020-05178-y.

2. Mao L, Jin H, Wang M, Hu Y, Chen S, He Q, et al. Neurologic Manifestations of Hospitalized Patients with Coronavirus Disease 2019 in Wuhan, China. JAMA Neurol. 2020 Jun 1;77(6):683-90. https://doi.org/10.1001/jamaneurol.2020.1127.

3. Yong SJ. Persistent Brainstem Dysfunction in Long-COVID: A Hypothesis. ACS Chem Neurosci. 2021 Feb 4. https://doi.org/10.1021/acschemneuro.0c00793.

4. The Lancet. Facing up to long COVID. Lancet. 2020;396(10266):1861. https://doi.org/10.1016/S0140-6736(20)32662-3.

5. Meeting the challenge of long COVID. Nat Med. 2020;26(12):1803. https://doi.org/10.1038/s41591020-01177-6.

6. Petersen MS, Kristiansen MF, Hanusson KD, Danielsen ME, á Steig B, Gaini S, et al. Long COVID in the Faroe Islands: A Longitudinal Study Among Nonhospitalized Patients. Clin Infect Dis Published online November 30, 2020. https://doi.org/10.1093/cid/ciaa1792.

7. Goërtz YMJ, Van Herck M, Delbressine JM, Vaes AW, Meys R, Machado FVC, et al. Persistent symptoms 3 months after a SARS-CoV-2 infection: the post-COVID-19 syndrome? ERJ Open Res. 2020;6(4):00542-2020. https://doi.org/10.1183/23120541.00542-2020.

8. Haute Autorité de Santé - Symptômes prolongés suite à une Covid-19 de l'adulte - Diagnostic et prise en charge. Accessed March 1. 2021. https://www.has-sante.fr/jcms/p_3237041/fr/symptomes- 
prolonges-suite-a-une-covid-19-de-l-adulte-diagnostic-et-prise-en-charge.

9. Excellence NI, for H, Practitioners C. RC of G, SIGN HIS. COVID-19 rapid guideline: managing the longterm effects of COVID-19. National Institute for Health and Care Excellence, London; 2020.

10. Sivan M, Taylor S. NICE guideline on long covid: Research must be done urgently to fill the many gaps in this new "living guideline." BMJ. 2020;371. http://doi.org/10.1136/bmj.m4938.

11. Tang YW, Schmitz JE, Persing DH, Stratton CW. Laboratory diagnosis of COVID-19: Current issues and challenges. J Clin Microbiol. 2020;58(6). https://doi.org/10.1128/JCM.00512-20.

12. Woloshin S, Patel N, Kesselheim AS. False Negative Tests for SARS-CoV-2 Infection - Challenges and Implications. N Engl J Med. 2020;383(6):e38. https://doi.org/10.1056/NEJMp2015897.

13. Cendejas-Bueno E, Romero-Gómez MP, Escosa L, Jiménez-Rodríguez S, Mingorance J, GarcíaRodríguez J, SARS-CoV-2 Working Group. Lower nasopharyngeal viral loads in pediatric population. The missing piece to understand SARS-CoV-2 infection in children? J Infect. 2021;S01634453(21)00289-9. https://doi.org/10.1016/j.jinf.2021.06.009.

14. Weisberg SP, Connors TJ, Zhu Y, et al. Distinct antibody responses to SARS-CoV-2 in children and adults across the COVID-19 clinical spectrum. Nat Immunol. 2021;22(1):25-31. https://doi.org/10.1038/s41590-020-00826-9.

15. Guedj E, Million M, Dudouet P, Tissot-Dupont $H$, Bregeon F, Cammilleri S, et al. 18F-FDG brain PET hypometabolism in post-SARS-CoV-2 infection: substrate for persistent/delayed disorders? Eur J Nucl Med Mol Imaging. 2021;1(2):592-5. https://doi.org/10.1007/s00259-020-04973-x. 48 ) .

16. Guedj E, Campion JY, Dudouet P, Kaphan E, Bregeon F, Tissot-Dupont H, et al. 18F-FDG brain PET hypometabolism in patients with long COVID. Eur J Nucl Med Mol Imaging Published online. 2021. https://doi.org/10.1007/s00259-021-05215-4.

17. Guedj E, Lazarini F, Morbelli S, Ceccaldi M, Hautefort C, Kas A, et al. Long COVID and the brain network of Proust's madeleine: targeting the olfactory pathway. Clin Microbiol Infect. 2021;S1198743X(21)00238-X. https://doi.org/10.1016/j.cmi.2021.05.015.

18. Ludvigsson JF. Case report and systematic review suggest that children may experience similar longterm effects to adults after clinical COVID-19. Acta Paediatr Int J Paediatr. 2020;110(3). https://doi.org/10.1111/apa.15673.

19. Smane L, Stars I, Pucuka Z, Roge I, Pavare J. Persistent clinical features in paediatric patients after SARS-CoV-2 virological recovery: A retrospective population-based cohort study from a single centre in Latvia. BMJ Paediatr Open. 2020;4(1):905. https://doi.org/10.1136/bmjpo-2020-000905.

20. Say D, Crawford N, McNab S, Wurzel D, Steer A, Tosif S. Post-acute COVID-19 outcomes in children with mild and asymptomatic disease. Lancet Child Adolesc Heal. 2021;5(6):e22-3. https://doi.org/10.1016/S2352-4642(21)00124-3.

21. Buonsenso D, Munblit D, De Rose C, Sinatti D, Ricchiuto A, Carfi A, et al. Preliminary Evidence on Long COVID in children. Acta Paediatr. 2021:110(7)2208-2211. https://doi.org/10.1111/apa.15870.

22. Molteni E, Sudre CH, Canas LS, Bhopal SS, Hughes RC, Antonelli M, et al. Illness duration and symptom profile in a large cohort of symptomatic UK school-aged children tested for SARS-CoV-2. 
medRxiv. 2021.

05.05.21256649

. https://doi.org/10.1101/2021.05.05.21256649.

23. Lagarde S, Lepine A, Caietta E, Pelletier F, Boucraut J, Chabrol B, et al. Cerebral 18FluoroDeoxyGlucose Positron Emission Tomography in paediatric anti N-methyl-D-aspartate receptor encephalitis: A case series. Brain Dev. 2016;38(5):461-70. https://doi.org/10.1016/j.braindev.2015.10.013.

24. Dong Y, Dong Y, Mo X, Hu Y, Qi X, Jiang F, et al. Epidemiology of COVID-19 among children in China. Pediatrics. 2020;145(6). https://doi.org/10.1542/peds.2020-0702.

25. Tzourio-Mazoyer N, Landeau B, Papathanassiou D, Crivello F, Etard O, Delcroix N,et al. Automated anatomical labeling of activations in SPM using a macroscopic anatomical parcellation of the MNI MRI single-subject brain. Neuroimage. 2002;15(1):273-89. https://doi.org/10.1006/nimg.2001.0978.

26. Lu Y, Li X, Geng D, Mei N, Wu P-Y, Huang C-C, et al. Cerebral Micro-Structural Changes in COVID-19 Patients - An MRI-based 3-month Follow-up Study. EClinicalMedicine. 2020;25:100484. https://doi.org/10.1016/j.eclinm.2020.100484.

27. Serrano GE, Walker JE, Arce R, Glass MJ, Vargas D, Sue LI, et al. Mapping of SARS-CoV-2 Brain Invasion and Histopathology in COVID-19 Disease. medRxiv Prepr Serv Heal Sci Published online February 18, 2021. https://doi.org/10.1101/2021.02.15.21251511.

28. Song E, Zhang C, Israelow B, Lu-Culligan A, Prado AV, Skriabine S, et al. Neuroinvasion of SARS-CoV2 in human and mouse brain. J Exp Med. 2021;218(3). https://doi.org/10.1084/jem.20202135.

29. Guedj E, Morbelli S, Kaphan E, Campion JY, Dudouet P, Ceccaldi M, et al. From early limbic inflammation to long COVID sequelae. Brain. 2021;awab215. https://doi.org/10.1093/brain/awab215.

30. Meinhardt J, Radke J, Dittmayer C, Franz J, Thomas C, Mothes R, et al. Olfactory transmucosal SARS-CoV-2 invasion as a port of central nervous system entry in individuals with COVID-19. Nat Neurosci. 2021;24(2):168-75. https://doi.org/10.1038/s41593-020-00758-5.

31. Bryche B, St Albin A, Murri S, Lacôte S, Pulido C, Ar Gouilh M, et al. Massive transient damage of the olfactory epithelium associated with infection of sustentacular cells by SARS-CoV-2 in golden Syrian hamsters. Brain Behav Immun. 2020;89:579-86. https://doi.org/10.1016/j.bbi.2020.06.032.

32. Niesen M, Trotta N, Noel A, Coolen T, Fayad G, Leurkin-Sterk G, et al. Structural and metabolic brain abnormalities in COVID-19 patients with sudden loss of smell. Eur J Nucl Med Mol Imaging. 2021;48:1890-901. https://doi.org/10.1007/s00259-020-05154-6.

33. Yuan MY, Chen ZK, Ni J, Wang TX, Jiang SY, Dong H, et al. Ablation of olfactory bulb glutamatergic neurons induces depressive-like behaviors and sleep disturbances in mice. Psychopharmacology. 2020;237(8):2517-30. https://doi.org/10.1007/s00213-020-05552-6.

34. Guilmot A, Maldonado Slootjes S, Sellimi A, Bronchain M, Hanseeuw B, Belkhir L, et al. Immunemediated neurological syndromes in SARS-CoV-2-infected patients. J Neurol. 2021;268:751-7. https://doi.org/10.1007/s00415-020-10108-x. 
35. Yeoh YK, Zuo T, Lui GCY, Zhang F, Liu Q, Li AYL, et al. Gut microbiota composition reflects disease severity and dysfunctional immune responses in patients with COVID-19. Gut. 2021;70(4). https://doi.org/10.1136/gutjnl-2020-323020.

36. Muzik O, Chugani DC, Juhász C, Shen C, Chugani HT. Statistical parametric mapping: Assessment of application in children. Neuroimage. 2000;12:538-49. https://doi.org/10.1006/nimg.2000.0651.

\section{Figures}

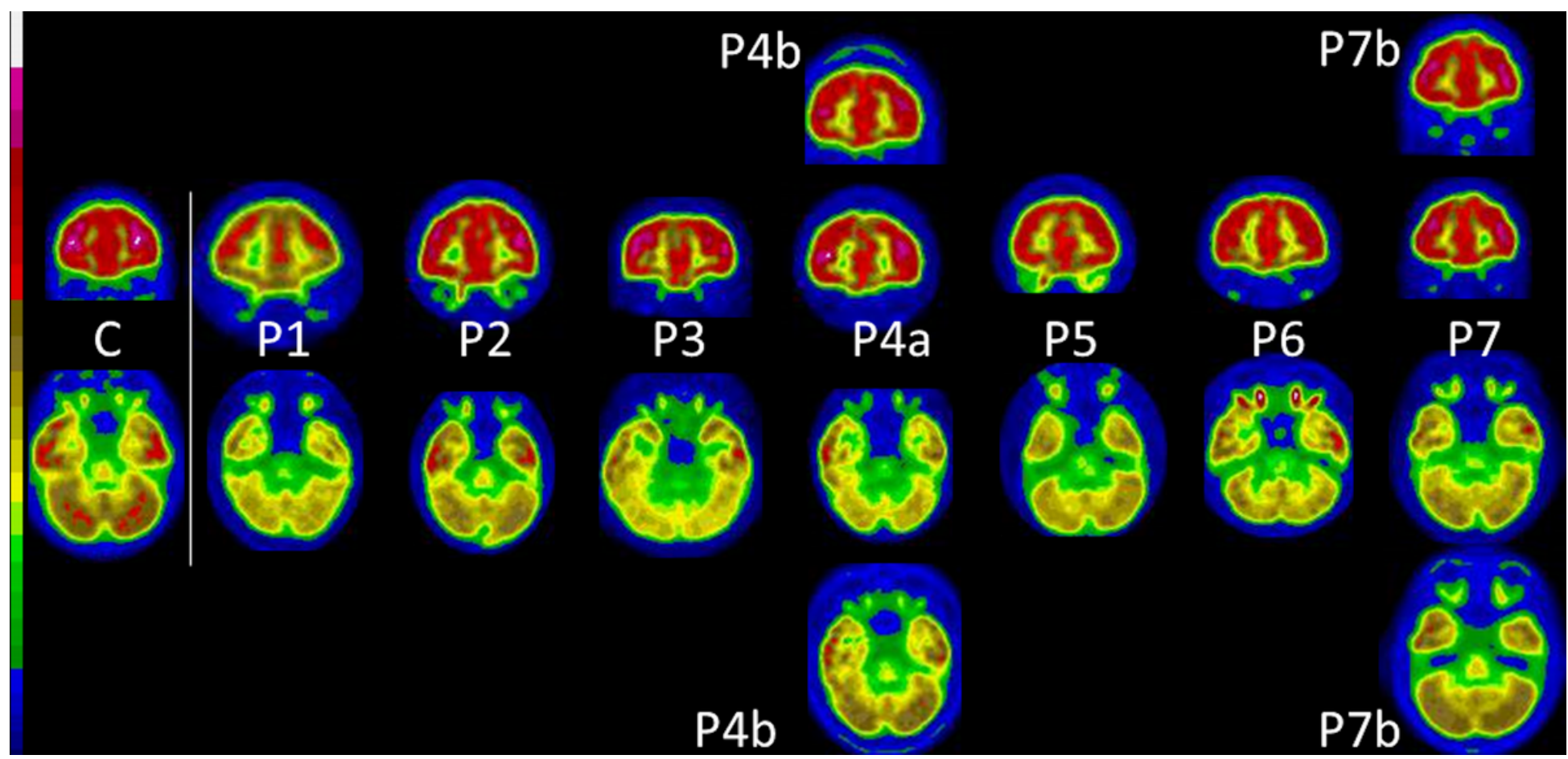

Figure 1

Individual 18F-FDG PET of each of the seven children patient (\#1 to \#7), including the follow-up of two of them (\#4b and \#7b) An example of normal PET metabolism in a child of 10 years old is also presented. Hypometabolism is found in olfactory regions for children $\# 1,3,4,5,6$; in temporal regions for children $\# 1,3,4,5,6,7$; in the brainstem for children $\# 1,3,4,5,6,7$; in the cerebellum for all children. At follow-up, the brain metabolism was improved at least for the brainstem. 


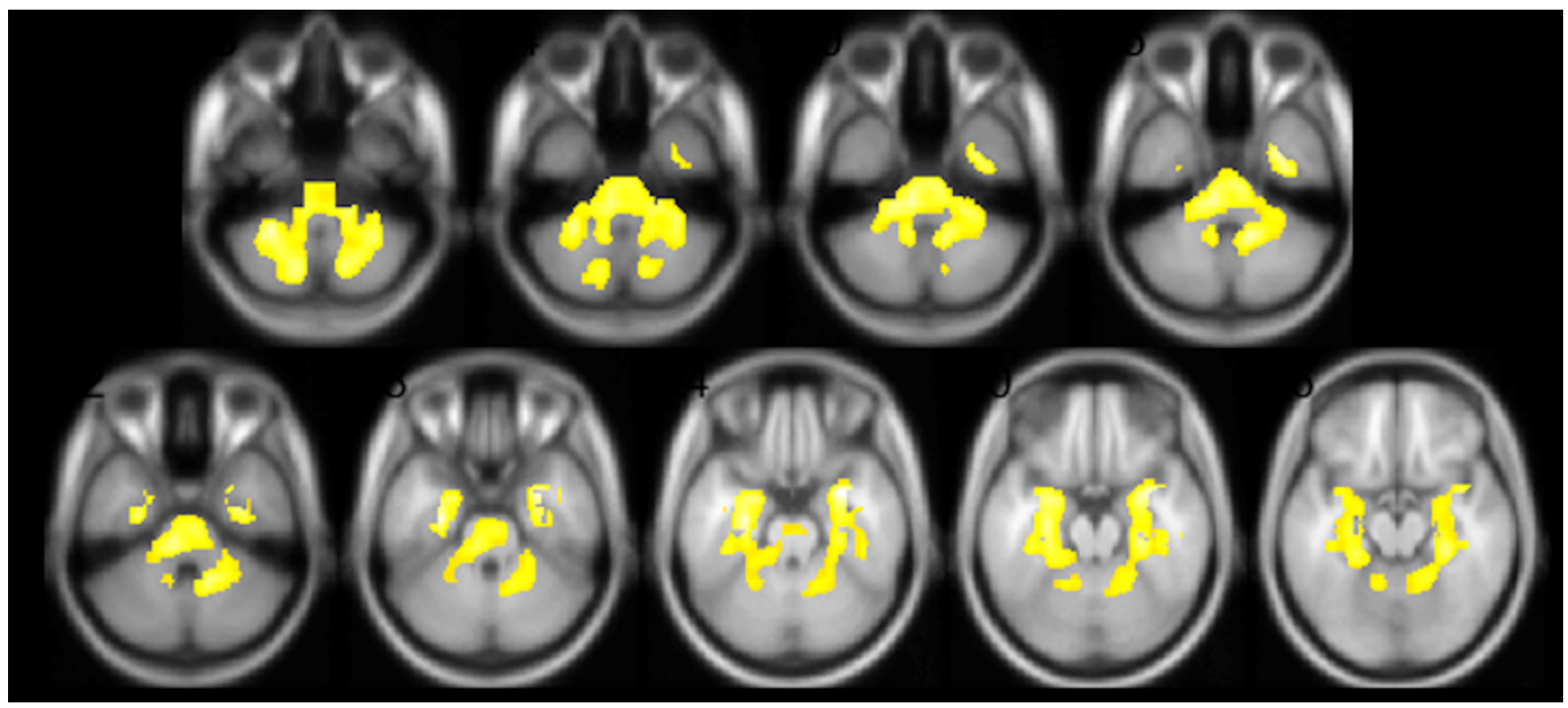

Figure 2

Brain 18F-FDG PET hypometabolism in paediatric patients with long COVID In comparison to paediatric controls, children with long COVID exhibited hypometabolism in bilateral medial lobes, the pons and cerebellum ( $p$-voxel $<0.001$, p-cluster $<0.05$ FWE-corrected), and also in the right olfactory gyrus after small volume correction ( $p$-voxel $=0.010 \mathrm{FWE}$-corrected). Findings are presented in axial MR slices (the left hemisphere is on the left side, anatomical convention).

\section{Supplementary Files}

This is a list of supplementary files associated with this preprint. Click to download.

- SupplementaryTable1.docx 